\section{(c) $(1)(9)$}

Revista EIA

ISSN 1794-1237

e-ISSN 2463-0950

Año XVIII/ Volumen 18/ Edición N.35

Enero-Junio de 2021

Reia35006 pp. 1-13

Publicación científica semestral Universidad EIA, Envigado, Colombia

\section{Para citar este artículo /} To REFERENCE THIS ARTICLE /

Maureira Cid, F.; Díaz-Muñoz, H.; Hadweh-Briceño, M.; Flores-Ferro, E.; Silva-Salse, A. (2021). Estabilidad de correlaciones de la actividad eléctrica no-lineal del cerebro en reposo con ojos cerrados.

Revista EIA, 18(35), Reia35006. pp. 1-13.

https://doi.org/10.24050/reia. v18i35.1463

\section{Autor de correspondencia:}

Maureira Cid, F. (Fernando): Docente de Neurociencia, Universidad Metropolitana de Ciencias de la Educación de Chile Correo electrónico: maureirafernando@yahoo.es

Recibido: 14-08-2020

Aceptado: 11-12-2020

Disponible online: 01-01-2021

\title{
Estabilidad de correlaciones de la actividad eléctrica no-lineal del cerebro en reposo con ojos cerrados
}

$\checkmark$ FERNANDO MAUREIRA CID ${ }^{1}$

HERNÁN DÍAZ-MuÑOZ ${ }^{2}$

MARCELO HADWEH-BRICEÑO ${ }^{3}$

ELIZABETH FLORES-FERRO ${ }^{4}$

ÁNGELA SILVA-SALSE ${ }^{5}$

1. Universidad Metropolitana de Ciencias de la Educación de Chile

2. Universidad de Santiago de Chile

3. Universidad Arturo Prat

4. Universidad Bernardo O'Higgins

5. Universidad Católica del Maule

\section{Resumen}

Introducción: la señal del EEG suele interpretarse desde una mirada lineal, sin embargo, desde hace algunas décadas se estudia la actividad eléctrica cerebral como un sistema dinámico, basado en la teoría del caos, con matemáticas no lineales. Objetivo: analizar la estabilidad de las correlaciones de los índices de Hurst a través del tiempo en sujetos en reposo con los ojos cerrados. Métodos: se evaluaron 13 varones universitarios con el dispositivo cerebro-interfaz Emotiv Epoc® con frecuencia de muestreo de $128 \mathrm{~Hz}$. Se analizaron los rangos de frecuencia delta $(1-3 \mathrm{~Hz})$, theta $(3,5-7 \mathrm{~Hz})$, alfa $(8-12 \mathrm{~Hz})$, beta $(13-30 \mathrm{~Hz})$ y gamma ( $>30 \mathrm{~Hz}$ ). Resultados: los resultados muestran estabilidad en el porcentaje de correlaciones en todas las bandas estudiadas en la mayoría de los sujetos. esta situación ocurre en ventanas temporales de 10, 30 y 60 segundos. Conclusiones: este estudio exploratorio muestra la persistencia en el tiempo de procesos meta-sincrónicos no-lineales que obedecen a la dinámica del balance caos/orden global del cerebro, en condiciones de reposo, basal con ojos cerrados.

Palabras Claves: electroencefalografía, exponente de Hurst, correlaciones, estado basal.

\section{Stability of correlations of non-linear electrical activity of the resting brain with closed eyes}




\begin{abstract}
Introduction: the signal of the EEG is usually interpreted from a linear perspective, however, for some decades now the electrical activity of the brain has been studied as a dynamic system, based on the theory of chaos, with non-linear mathematics. Objective: analize the stability of correlations of hurst indices over time in resting subjects with closed eyes. Methods: 13 male university students were evaluated with the brain-interface device Emotiv Epoc® with sampling frequency of $128 \mathrm{~Hz}$. the frequency ranges delta (1-3 Hz), theta (3.5-7 Hz), alpha (8-12 $\mathrm{Hz})$, beta $(13-30 \mathrm{~Hz})$ and gamma $(>30 \mathrm{~Hz})$ were analyzed. Results: the results show stability in the percentage of correlations in all the bands studied in most of the subjects. this situation occurs in temporary windows of 10, 30 and 60 seconds. Conclusion: this exploratory study shows the persistence intime of non-linear meta-synchronous processes that obey the dynamics of balance chaos/global order of the brain, in resting conditions, basal with closed eyes.
\end{abstract}

Key Words: elecroencephalohraphy, hurst index, correlations, baseline status.

\title{
1. Introducción
}

En 1929 Hans Berger publicó los primeros registros de la actividad eléctrica cerebral de seres humanos (F. Maureira; 2017), desde entonces se ha utilizado la electroencefalografía (EEG) para el estudio del cerebro en estados patológicos y en décadas recientes, para comprender qué cambios en la actividad eléctrica subyacen a diversos procesos motores, sensoriales y cognitivos (C. Michel, M. Murray; 2011). Los registros del EEG entregan cinco tipos de ondas cerebrales asociados a diversos procesos: a) onda alfa ( $8-12 \mathrm{~Hz}$ ) presente cuando un sujeto está despierto, relajado y con los ojos cerrados; b) onda beta $(13-30 \mathrm{~Hz})$ característica de un sujeto despierto y realizando actividades perceptuales y cognitivas; c) onda gamma $(>30 \mathrm{~Hz})$ presente en estados de meditación y altos niveles de concentración; d) onda theta (3.5-7 Hz) característica durante el sueño ligero; e) onda delta (1-3 Hz) característica durante el sueño profundo (M. Bear, B. Connors, M. Paradiso; 2016).

En forma clásica, la señal del EEG se ha interpretado desde una mirada lineal (F. Maureira, F. Flores; 2018), sin embargo, desde hace algunas décadas se estudia la actividad eléctrica cerebral como un sistema dinámico, basado en la teoría del caos, con matemáticas no lineales (F. Montero, F. Morán; 1992), donde pequeñas modificaciones de los parámetros iniciales pueden incidir en cambios de magnitud, haciendo imposible la predicción de su devenir futuro (H. Díaz, F. Córdova, et al; 2015, M; 2011) Corless. La dinámica que presenta la actividad eléctrica cerebral registrada a través de un EEG representa la confluencia de numerosas fuentes de variación simultánea en el tiempo y como tal, es un ejemplo de dinámica no-lineal compleja (J. Kumar, P. Bhuvaneswari; 2012).

El estudio de dinámicas no-lineales permite abordar la comprensión de fenómenos que no responden de manera proporcional frente a la presentación de un estímulo de intensidad creciente (A. Pikovsky, M. Rosenblum, J. Kurths; 2001) Fenómenos no-lineales simples son representados por curvas de crecimiento exponencial o logístico, en donde la respuesta de la variable independiente no progresa de manera lineal o proporcional y depende de determinados rangos de intensidad de la variable dependiente. Fenómenos no-lineales más complejos o multifactoriales no son posibles de graficar de manera sencilla ya que dependen de numerosas variables que, a su vez, pueden responder a dinámicas lineales o nolineales propias, haciendo imposible abordar estos fenómenos de manera totalmente predictiva (M. Corless; 2011). 
Con el fin de abordar estos fenómenos, durante las últimas décadas se han implementado métodos que permiten dilucidar en parte la complejidad natural de estas dinámicas no-lineales, haciéndolas relativa o parcialmente predecibles, para lo cual se recurre a encontrar patrones regulares que se repiten en distintas escalas temporales (H. Díaz, F. Maureira, et al; 2019, H. Díaz, F. Maureira; 2015).

La derivación matemática realizada por Harold Edwin Hurst (1880-1978) para determinar los patrones de variabilidad en el tiempo de las lluvias y sequias que alimentan al Rio Nilo en Egipto, dio origen al exponente de Hurst, un indicador que evalúa el grado de predictibilidad de una variable en el tiempo. Esta predictibilidad dependerá del detalle, muchas veces desconocido, de la estructura interna del sistema compuesto de patrones regulares y estocásticos que ocurren en ella, y que aparecen a distintas escalas de tiempo (J. Bassingthwaighte, G. Raymond; 1994) El índice o exponente de Hurst es un estadístico no-lineal que varía entre 0 y 1 , con tres rangos de predictibilidad variable sobre la dinámica interna del fenómeno de estudio. Para valores de $\mathrm{H}$ (exponente de Hurst) entre 0 y 0,45 , se resuelven tipos de dinámicas denominadas anti-persistentes, es decir, oscilaciones en el tiempo que, si bien son caóticas, con bajo grado de predictibilidad, tienden a permanecer en torno a un determinado valor central de la variable, pero sin sugerir un comportamiento futuro. Valores en torno a un exponente de Hurst en el rango 0.45-0.55, son indicadores de dinámicas en donde la variable medida se comporta como un movimiento browniano, random-walk, o ruido café, es decir, una dinámica definida como ruido estadístico, sin contenido informacional relevante (M. Raimundo, J. Okamoto; 2018). Los valores del exponente de Hurst por sobre este último rango $(\mathrm{H}>0.55)$, y hasta el valor 1, representan el "efecto Hurst" y corresponden a sistemas que se alejan de la impredictibilidad, o del caos, y cuya dinámica interna está siendo gobernada por procesos con alto contenido informacional y que conducen el devenir de la variable de estudio a través de una cierta tendencia, un patrón creciente o decreciente, con probabilidad de permanecer en el tiempo (H. Díaz, F. Maureira, et al; 2018, G. Layek; 2015).

Los registros EEG en condición de reposo basal con los ojos cerrados se constituyen como referencias para protocolos experimentales, estudiando la variación de la actividad cerebral antes y después de una intervención (G. Buzsaki; 2006). Un estudio de correlaciones de índices de Hurst de ondas beta entre diversas regiones cerebrales muestra que al pasar de un estado de reposo a un trabajo cognitivo de alta demanda (resolución de problemas) disminuye el porcentaje de correlaciones entre pares de electrodos, lo que fue explicado por la necesidad de un trabajo cerebral más independiente, donde cada región se encarga de resolver un ámbito específico del problema, lo que lleva a la desincronización global de la actividad cerebral (H. Díaz, F. Maureira, et al; 2019). También se ha observado una mayor correlación de índices $\mathrm{H}$ entre electrodos inter-hemisféricos, revelando mayor sincronización, en estado de reposo con los ojos cerrados, entre regiones del hemisferio derecho e izquierdo, que dentro de un mismo hemisferio. Esta situación ocurre en las ondas delta, theta, alfa y beta (H. Díaz, F. Maureira, et al; 2019).

Siguiendo con análisis de índices $\mathrm{H}$ en señales de EEG, otro estudio muestra que no existen diferencias en el número de pares de electrodos relacionados en condiciones de ojos abiertos y cerrados (H. Díaz, F. Maureira; 2018), y que los pares de electrodos altamente correlacionados permanecen estables en dichas relaciones cuando el sujeto pasa de una condición de reposo con ojos cerrados a ojos abiertos, pero con un estado más caótico e impredecible en el eje fronto-occipital en el primer estado y más equilibrado con los ojos abiertos (H. Díaz, F. Maureira, F. Córdova; 2018). 
El estudio de la actividad y la relación de las diversas regiones cerebrales bajo el paradigma de sistema caóticos es reciente, situación que da cuenta de la necesidad de profundizar en estos fenómenos y dar cuenta de la actividad eléctrica registrada con el EEG como un sistema dinámico. Debido a lo anterior, es que surge el objetivo de la presente investigación: analizar si el número (o porcentaje) de correlaciones de series de tiempo compuestas de índices de Hurst, extraídos cada 1 segundo a partir de 6 canales EEG, durante un estado de reposo con ojos cerrados, se mantiene estable durante diferentes ventanas temporales dentro de los 2 minutos de registro electroencefalográfico, en condiciones basales con los ojos cerrados.

\section{Métodos}

Se trabajó con una muestra no probabilística intencionada. Se evaluaron a 13 estudiantes varones de educación física de una Universidad privada de Santiago de Chile, con edad promedio de $19.1 \pm 1.43$ años. Para formar parte de la muestra los participantes no debían presentaba alteraciones cognitivas, consumir medicamentos y tampoco estar en tratamiento psicológico a lo menos un año antes de las evaluaciones. Durante el presente estudio se respetaron los principios éticos para las investigaciones médicas con seres humanos de la Declaración de Helsinki (World Medical Asociation; 2013). Esta investigación fue aprobada por el comité de ética de la Universidad Católica Silva Henríquez mediante el acta s/n de mayo de 2018. Todos los participantes firmaron un consentimiento informado.

Para el registro EEG se utilizó el dispositivo cerebro-interfaz Emotiv Epoc® con frecuencia de muestreo de $128 \mathrm{~Hz}$. El EEG registra 14 canales a través de electrodos posicionados según el sistema 10/20 (Fig. 1) usando como referencia los electrodos del hueso mastoides. Los datos del EEG fueron procesados con el programa EEGLAB y ADJUST ejecutados en la plataforma MATLAB 2008. Se utilizaron los registros del lóbulo frontal (AF3 y AF4), temporal (T7 y T8) y occipital (01 y 02).

Figura 1. Localización de los electrodos del Emotiv Epoc ${ }^{\circledR}$ durante el estudio.

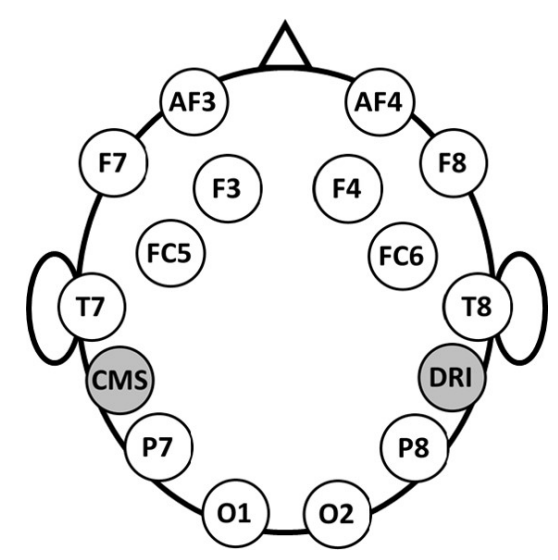

Se registró la frecuencia cardíaca basal de cada participante durante cinco minutos sentado relajadamente en una silla. Posterior a ello, se realizó un registro basal de la actividad cerebral con dos minutos con los ojos cerrados y se analizó el rango de frecuencia delta $(1-3 \mathrm{~Hz})$, theta $(3.5-7 \mathrm{~Hz})$, alfa $(8-12 \mathrm{~Hz})$, beta $(13-30 \mathrm{~Hz})$ y gamma 
$(>30 \mathrm{~Hz}$ ). Todas las mediciones se realizaron durante la mañana (entre 10:00 y 12:00 hrs.). Previo a este registro a cada participante se le entregaron indicaciones sobre sueño, consumo de alcohol y bebidas energizantes, para el día previo a la evaluación. Es importante señalar que la totalidad de los participantes declararon no consumir drogas de forma habitual

Para el registro y análisis de datos del electroencefalograma se utilizó el programa Matlab con la herramienta EEGLAB. Posteriormente, se procedió a calcular el exponente de Hurst, el cual se puede aplicar a series de tiempo de la actividad eléctrica cerebral humana, o EEG, en este caso en un estado basal con los ojos cerrados durante 2 minutos. Este procedimiento arroja un valor que estima el balance caos/orden de la señal EEG (H. Díaz, F. Maureira; 2015)

En una segunda derivación de este método (H. Díaz, F. Maureira; 2019) dividimos la serie original de tiempo de 2 minutos, en segmentos de 1 segundo de duración cada uno (128 valores de la serie EEG original muestreados a $128 \mathrm{~Hz}$ ), de esta manera obtuvimos una nueva serie de tiempo con 120 valores de Hurst, obtenidos uno por cada segundo de la serie original de 2 minutos. Luego, volvimos a aplicar el exponente de Hurst sobre esta nueva serie derivada de 120 valores de Hurst. Este método, a diferencia de la definición original del exponente de Hurst, evalúa ahora el balance caos/ orden de la serie de tiempo que representa la oscilación de este balance caos/orden en el tiempo, es decir, permite estimar cuán predecible o irregular, es la tendencia caótica del balance caos/orden de la serie de tiempo derivada de valores de Hurst y que denominamos m-Hurst o HH (20).

En un trabajo reciente (H. Díaz, F. Maureira; 2019), reportamos la posibilidad de encontrar cierto grado de correlación de esta serie temporal m-Hurst entre pares de canales EEG, sugiriendo que la estabilidad/inestabilidad de esta oscilación metacaótica podría contener cierto significado biológico para el balance de las dinámicas cerebrales inter e intra-hemisféricas.

Se calcularon los índices de m-Hurst de los segundos 1 al 60 y del 61 al 120 . También de los segundos 1 al 30, 31 al 60, 61 al 90 y 91 al 120, y finalmente, cada 10 segundos. Esto se realizó para cada uno de los seis electrodos (AF3, AF4, T7, T8, 01 y 02) de cada sujeto evaluado. Una vez determinadas las medias de los índices $\mathrm{H}$ se utilizó el programa estadístico SPSS 25.0 para Windows. La prueba de KolmogorovSmirnov (KS) no entregó una distribución normal para los datos analizados $(\mathrm{p}<0.05)$ razón por la cual se procedió a utilizar estadística no paramétrica. Para relacionar los índices $\mathrm{H}$ entre los seis electrodos estudiados en cada ventana temporal se utilizaron correlaciones Spearman, para comparar el número de correlaciones de cada electrodo entre el primer y segundo minuto de registro se utilizó la prueba de Wilcoxon, y para comparar el número de correlaciones de cada electrodo entre los cuatro períodos de 30 segundos y entre los 12 períodos de 10 segundos se utilizó la prueba de Friedmann. Se consideraron significativos valores $\mathrm{p}<0.05$.

\section{Resultados}

A continuación, se presentan los resultados del número de correlaciones de la serie temporal de índices de Hurst entre los seis electrodos estudiados en diferentes ventanas temporales. En la figura 2 se observa un ejemplo de las correlaciones superiores a 0.600 del sujeto 1 de la muestra. Es posible notar que, durante períodos de 60 segundos, el número de correlaciones permanecen bastante estables. Durante el primer minuto del sujeto en estado de reposo con los ojos cerrados, la onda delta presenta 
correlaciones entre la región prefrontal derecho (AF4) y la región temporal derecho (T8), también existe relación entre la región temporal derecho (T8) y la región occipital derecho (02). Esto representa el 13.3\% de las correlaciones posibles entre los seis electrodos utilizados para los análisis. La misma situación vuelve a ocurrir durante el segundo minuto de registro de EEG. Es posible notar que las ondas gamma presentan un aumento de $0 \%$ a $6.7 \%$ de correlaciones durante el segundo minuto.

Figura 2. Correlaciones mayores a 0,600 en períodos de 60 segundos en las cinco ondas estudiadas. Se describe el sujeto 1 de la muestra. AF3=prefrontal izquierdo; $\mathrm{AF} 4=$ prefrontal derecho; $\mathrm{T7}=$ temporal izquierdo; T8=temporal derecho; $\mathrm{O} 1=$ occipital izquierdo; $\mathrm{O} 2=$ occipital derecho.

Fuente: elaboración propia basada en los datos obtenidos en el estudio
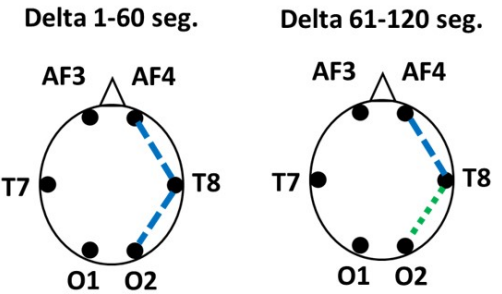

Theta 1-60 seg.

Theta 61-120 seg.
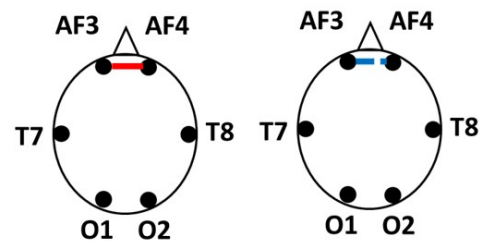

Alfa 1-60 seg.

Alfa 61-120 seg.

Beta 1-60 seg.

Beta 61-120 seg.
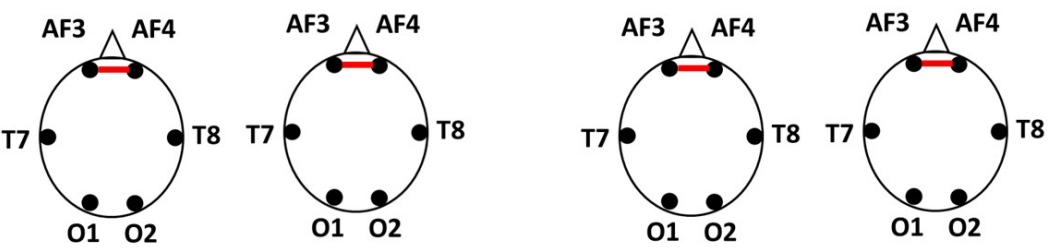

Gamma 1-60 seg. Gamma 61-120 seg.
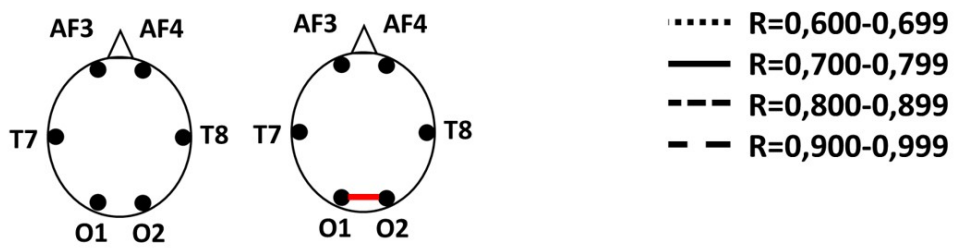

En la figura 3 se muestran las variaciones de los porcentajes de correlaciones de los índices $\mathrm{H}$ entre el primer y segundo minuto de registro del EEG. En la onda delta todos los sujetos (a excepción del sujeto 2) presentan entre $0 \%$ y 30\% de correlaciones entre las seis regiones cerebrales estudiadas, con cinco sujetos que no presentan variación entre ambos períodos de tiempo. La onda delta, durante los primeros 60 segundos, presenta una media de $12.3 \%$ de correlaciones, mientras que, en los siguientes 60 segundos, una media de $13.3 \%$ sin observarse diferencias significativas entre estos dos periodos $(\mathrm{p}=0.194)$.

La onda theta presenta correlaciones entre $0 \%$ y $20 \%$ en ambos períodos de tiempo, con ocho sujetos que presentan la misma cantidad de correlaciones (un 6.7\%) en los dos minutos de registro. Ambas ventanas temporales presentan una media de $6.2 \%$ de correlaciones $(\mathrm{p}=1.000)$. La onda alfa presenta un número de correlaciones 
que va entre $0 \%$ y $13.3 \%$ en ambos períodos de tiempo, con 10 sujetos que presentan un $6.7 \%$ de correlaciones en los minutos uno y dos. Existe una leve disminución del 7.2\%, durante los primeros 60 segundos, a un 5.7\% de correlaciones, durante el segundo minuto, sin que existan diferencias significativas entre estos valores $(\mathrm{p}=0.102)$.

La onda beta presenta entre $0 \%$ y $26.7 \%$ de correlaciones en los dos períodos de tiempo, con cuatro sujetos que presentan un $6.7 \%$ de correlaciones en ambos minutos. Existe una leve disminución de 5.1\% a 3.6\% de correlaciones entre el minuto uno $y$ dos, sin que exista diferencia significativa $(\mathrm{p}=0.257)$. La onda gamma presenta, en 12 sujetos, valores de $0 \%$ de correlaciones en ambos minutos $(\mathrm{p}=0.317)$.

Figura 3. Variaciones de los porcentajes de correlaciones de las cinco ondas cerebrales durante ventanas temporales de 60 segundos en todos los sujetos de la muestra. En algunos gráficos no se observan las líneas de todos los sujetos evaluados debido a que varios de ellos coinciden en los porcentajes en ambos períodos de tiempo.

Fuente: elaboración propia basada en los datos obtenidos en el estudio
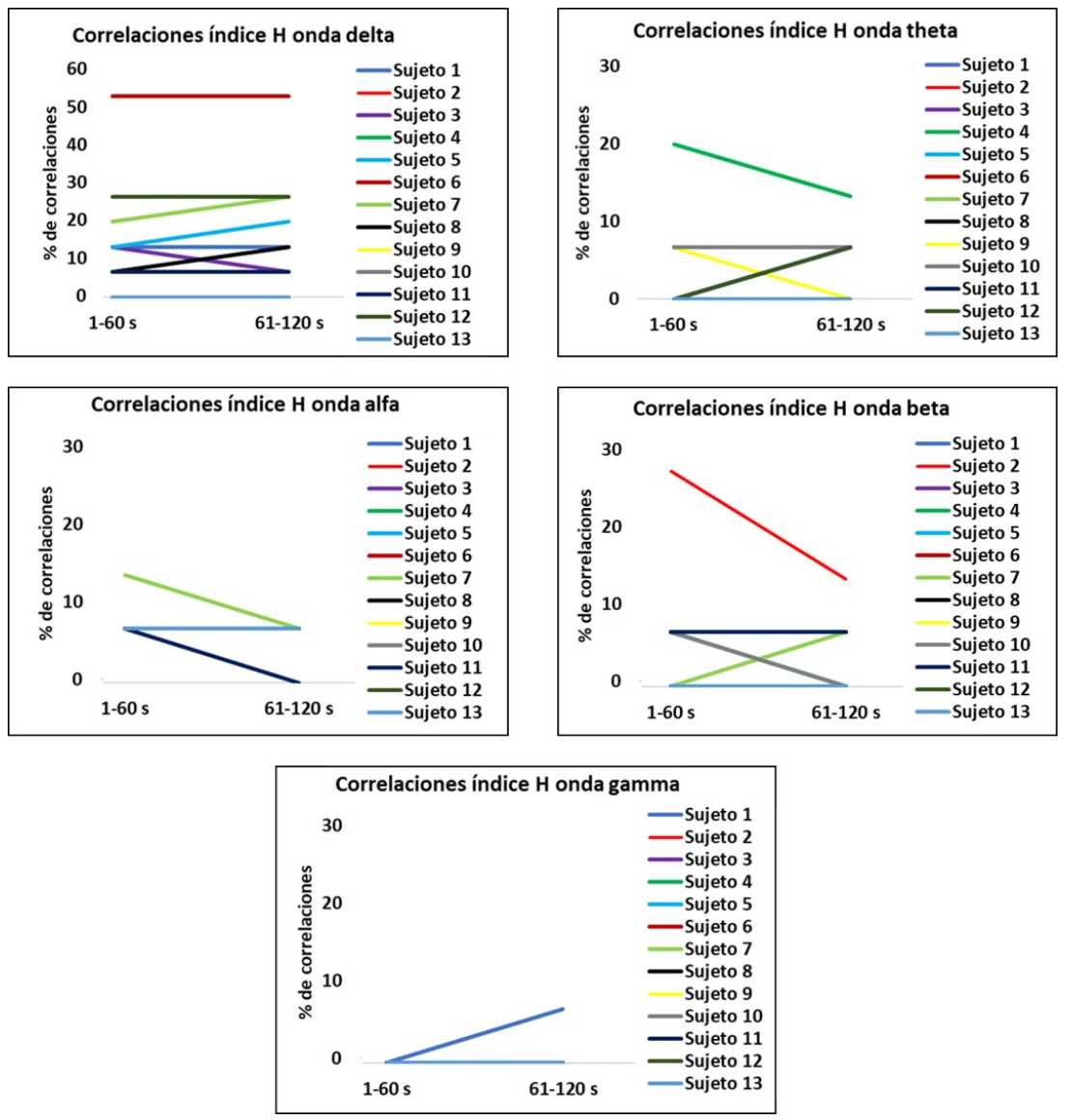

En la figura 4 se muestran las variaciones de los porcentajes de correlaciones de los índices $\mathrm{H}$ en ventanas temporales de 30 segundos $(\mathrm{t} 1=1-30 \mathrm{~s}, \mathrm{t} 2=31-60 \mathrm{~s}, \mathrm{t} 3=61$ $90 \mathrm{~s}$ y $\mathrm{t} 4=91-120 \mathrm{~s}$ ) de registro del EEG. En la onda delta todos los sujetos evaluados (excepto el sujeto 2) presentan entre 0\% y 30\% de correlaciones en los cuatro perío- 
dos de tiempo. Las medias de porcentajes de correlaciones fueron: $\mathrm{t} 1=14.5 ; \mathrm{t} 2=13.3$; $\mathrm{t} 3=13.4 ; \mathrm{t} 4=14.4$ sin que existan diferencias significativas entre ellas ( $\mathrm{p}=0.546)$.

En la onda theta todos los sujetos evaluados presentan entre $0 \%$ y $33.3 \%$ de correlaciones en los cuatro períodos de tiempo. Las medias de porcentajes de correlaciones fueron: $\mathrm{t} 1=8.7 ; \mathrm{t} 2=6.7 ; \mathrm{t} 3=6.2 ; \mathrm{t} 4=7.7$ sin que existan diferencias significativas entre ellas $(\mathrm{p}=0.639)$. En la onda alfa todos los sujetos evaluados presentan entre $0 \%$ y $20 \%$ de correlaciones en los cuatro períodos de tiempo. Las medias de porcentajes de correlaciones fueron: $\mathrm{t} 1=6.7 ; \mathrm{t} 2=8.7 ; \mathrm{t} 3=6.2 ; \mathrm{t} 4=7.2$ sin que existan diferencias significativas entre ellas ( $\mathrm{p}=0.436$ ). En la onda beta todos los sujetos evaluados (excepto el sujeto 2) presentan entre $0 \%$ y $13.3 \%$ de correlaciones en los cuatro períodos de tiempo. Las medias de porcentajes de correlaciones fueron: $\mathrm{t} 1=5.7 ; \mathrm{t} 2=6.7 ; \mathrm{t} 3=2.6$; $\mathrm{t} 4=3.6$ sin que existan diferencias significativas entre ellas ( $\mathrm{p}=0.056)$. En la onda gamma todos los sujetos evaluados presentan entre $0 \%$ y $6.7 \%$ de correlaciones en los cuatro períodos de tiempo. Las medias de porcentajes de correlaciones fueron: $\mathrm{t} 1=0.0 ; \mathrm{t} 2=1.0 ; \mathrm{t} 3=2.6 ; \mathrm{t} 4=0.5$ sin que existan diferencias significativas entre ellas $(\mathrm{p}=0.054)$.

\section{Figura 4. Variaciones de los porcentajes de correlaciones de las cinco ondas cerebrales durante ventanas temporales de 30 segundos en todos los sujetos de la muestra. En algunos gráficos no se observan las líneas de todos los sujetos evalua- dos debido a que varios de ellos coinciden en los porcentajes en ambos períodos de tiempo.}

\section{Fuente: elaboración propia basada en los datos obtenidos en el estudio}
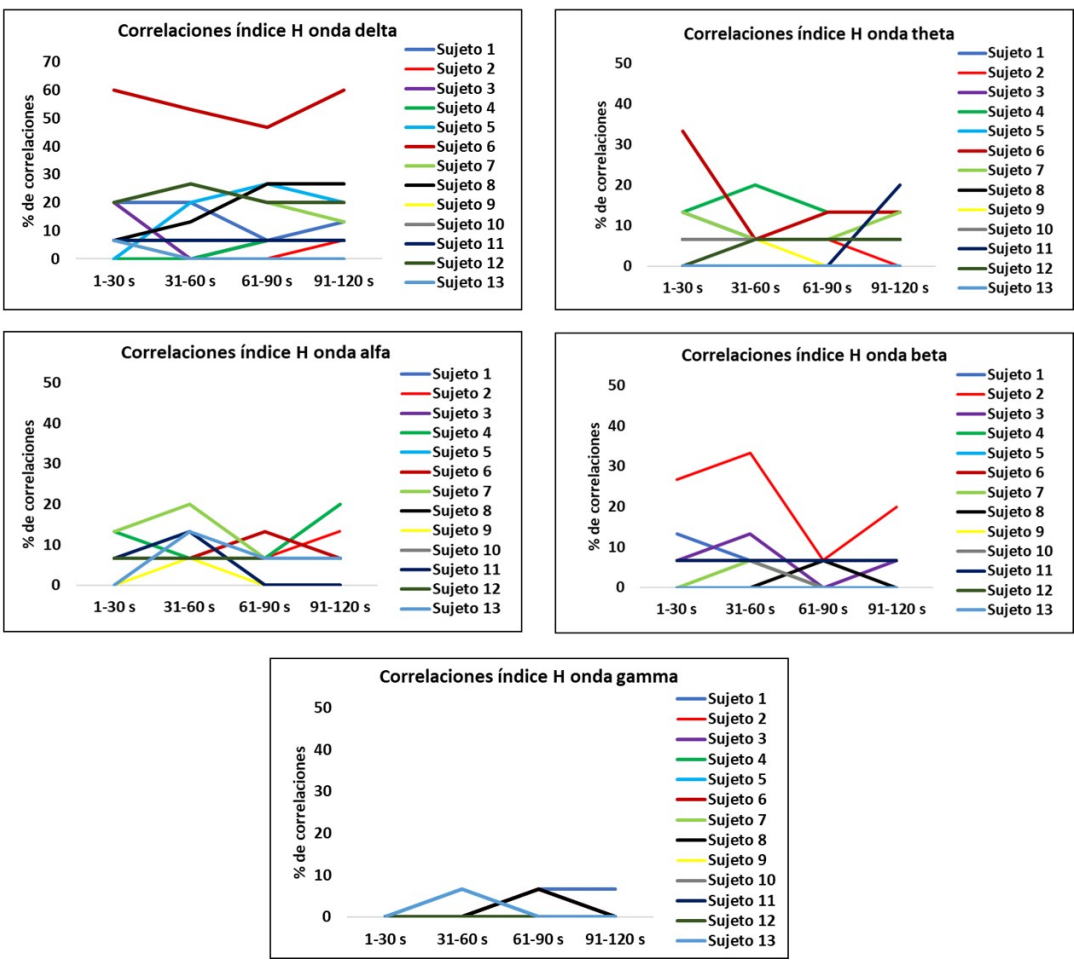

En la figura 5 se muestran las variaciones de los porcentajes de correlaciones de los índices $\mathrm{H}$ en ventanas temporales de 10 segundos $(\mathrm{t} 1=1-10 \mathrm{~s}, \mathrm{t} 2=11-20 \mathrm{~s}, \mathrm{t} 3=21$ $30 \mathrm{~s}, \mathrm{t} 4=31-40 \mathrm{~s}, \mathrm{t} 5=41-50 \mathrm{~s}, \mathrm{t} 6=51-60 \mathrm{~s}, \mathrm{t} 7=61-70 \mathrm{~s}, \mathrm{t} 8=71-80 \mathrm{~s}, \mathrm{t} 9=81-90 \mathrm{~s}, \mathrm{t} 10=91-$ 
$100 \mathrm{~s}, \mathrm{t} 11=101-110$ y t12=111-120 s) de registro del EEG. En la onda delta todos los sujetos evaluados (excepto el sujeto 2) presentan entre $0 \%$ y $50 \%$ de correlaciones en los doce períodos de tiempo. Las medias de porcentajes de correlaciones fueron: $\mathrm{t} 1=22.6 ; \mathrm{t} 2=19.5 ; \mathrm{t} 3=17.4 ; \mathrm{t} 4=18.3 ; \mathrm{t} 5=19.0 ; \mathrm{t} 6=22.6 ; \mathrm{t} 7=19.0 ; \mathrm{t} 8=19.0 ; \mathrm{t} 9=20.0$; $\mathrm{t} 10=15.9 ; \mathrm{t} 11=26.7 ; \mathrm{t} 12=18.5$ sin que existan diferencias significativas entre ellas $(\mathrm{p}=0.667)$.

En la onda theta todos los sujetos evaluados presentan entre $0 \%$ y $60 \%$ de correlaciones en los doce períodos de tiempo. Las medias de porcentajes de correlaciones fueron: $\mathrm{t} 1=16.2 ; \mathrm{t} 2=20.5 ; \mathrm{t} 3=15.9 ; \mathrm{t} 4=13.3 ; \mathrm{t} 5=11.8 ; \mathrm{t} 6=15.4 ; \mathrm{t} 7=13.3 ; \mathrm{t} 8=13.8$; $\mathrm{t} 9=13.8 ; \mathrm{t} 10=15.9 ; \mathrm{t} 11=14.9 ; \mathrm{t} 12=13.3$ sin que existan diferencias significativas entre ellas ( $\mathrm{p}=0.892)$. En la onda alfa todos los sujetos evaluados presentan entre $0 \%$ y $33.3 \%$ de correlaciones en los doce períodos de tiempo. Las medias de porcentajes de correlaciones fueron: $\mathrm{t} 1=12.8 ; \mathrm{t} 2=12.8 ; \mathrm{t} 3=15.9 ; \mathrm{t} 4=13.3 ; \mathrm{t} 5=10.8 ; \mathrm{t} 6=9.8 ; \mathrm{t} 7=12.8$; $\mathrm{t} 8=13.8 ; \mathrm{t} 9=14.4 ; \mathrm{t} 10=14.9 ; \mathrm{t} 11=9.2 ; \mathrm{t} 12=11.8$ sin que existan diferencias significativas entre ellas $(\mathrm{p}=0.154)$.

En la onda beta todos los sujetos evaluados presentan entre $0 \%$ y $50 \%$ de correlaciones en los doce períodos de tiempo. Las medias de porcentajes de correlaciones fueron: $\mathrm{t} 1=11.8 ; \mathrm{t} 2=11.3 ; \mathrm{t} 3=7.2 ; \mathrm{t} 4=10.3 ; \mathrm{t} 5=8.2 ; \mathrm{t} 6=9.0 ; \mathrm{t} 7=8.2 ; \mathrm{t} 8=7.7$; $\mathrm{t} 9=15.4 ; \mathrm{t} 10=10.8 ; \mathrm{t} 11=9.7 ; \mathrm{t} 12=9.8$ sin que existan diferencias significativas entre ellas $(\mathrm{p}=0.660)$. En la onda gamma todos los sujetos evaluados presentan entre $0 \% \mathrm{y}$ $40 \%$ de correlaciones en los doce períodos de tiempo. Las medias de porcentajes de correlaciones fueron: $\mathrm{t} 1=7.7 ; \mathrm{t} 2=2.6 ; \mathrm{t} 3=10.3 ; \mathrm{t} 4=6.2 ; \mathrm{t} 5=9.2 ; \mathrm{t} 6=9.2 ; \mathrm{t} 7=7.2 ; \mathrm{t} 8=10.8$; $\mathrm{t} 9=9.8 ; \mathrm{t} 10=6.2 ; \mathrm{t} 11=9.8 ; \mathrm{t} 12=7.7$ sin que existan diferencias significativas entre ellas $(\mathrm{p}=0.239)$.

Figura 5. Variaciones de los porcentajes de correlaciones de las cinco ondas cerebrales durante ventanas temporales de 10 segundos en todos los sujetos de la muestra.

Fuente: elaboración propia basada en los datos obtenidos en el estudio
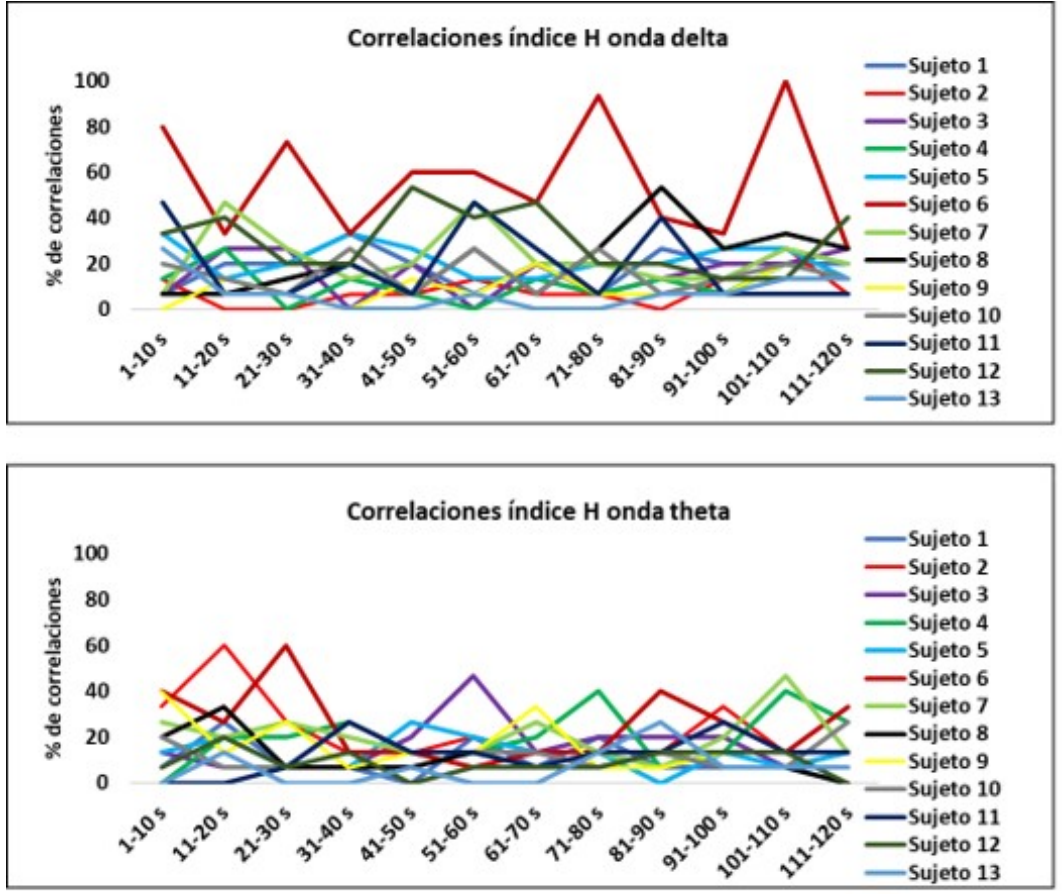


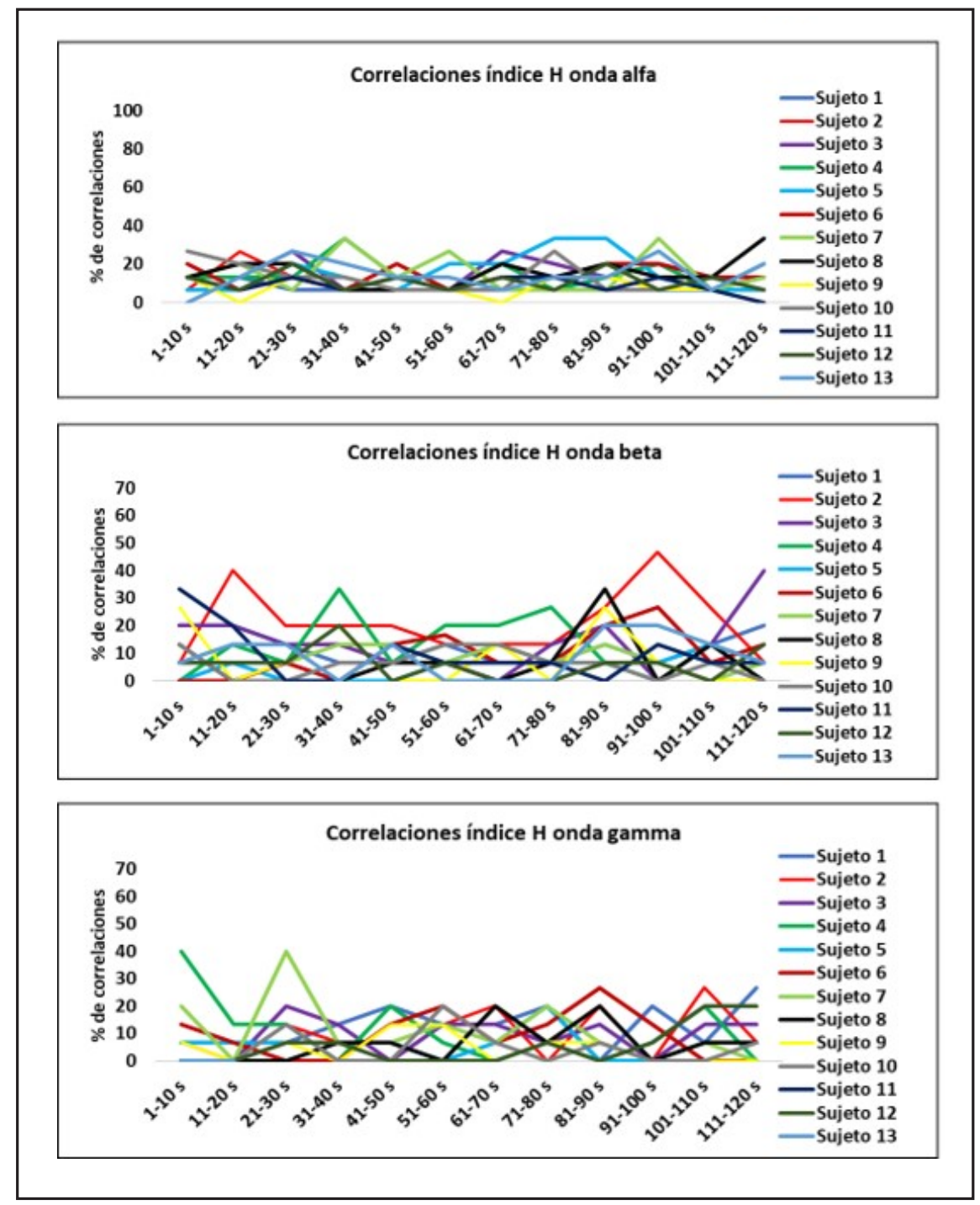

\section{Discusión}

El estudio de la dimensión no-lineal de los fenómenos cerebrales ha abierto la posibilidad de explorar la fenomenología y el comportamiento de la actividad electroencefalográfica desde una perspectiva que asume la imposibilidad de realizar una predicción cierta respecto del comportamiento futuro del sistema, pero que al mismo tiempo acepta la posibilidad de develar la existencia de patrones o tendencias crípticas, escondidas bajo la operación simultánea de procesos que se desarrollan a distintas escalas de tiempo y que, en su conjunto, definen un cierto estado de posibilidades de variación dirigida, o estabilidad futura.

En este sentido, el índice de Hurst evalúa estas tendencias a través de un estudio re-escalado de la serie de tiempo original (en este caso una serie de datos EEG), con el fin de poner de manifiesto ciertas predisposiciones del operar del sistema, que especifican dominios probabilísticos de sus tendencias futuras. 
En una segunda derivada de este principio rector, la presente investigación se centró en estudiar la variación en el tiempo de esta oscilación caótica expresada como una serie temporal de valores de Hurst, extraídos cada 1 segundo, a partir de la serie original de datos EEG. Con esto, se obtuvo una serie de tiempo de Hurst de 120 datos correspondientes a 2 minutos de registro inicial del EEG, en condiciones de reposo basal con los ojos cerrados, y se procedió a correlacionar estas series de tiempo de Hurst entre los 6 canales EEG que se escogieron para este estudio (AF3, AF4, T7, T8, 01, y 02).

Los resultados muestran que el porcentaje de pares de electrodos altamente correlacionados $(\mathrm{R}>0.600)$ para series de tiempo de Hurst, alcanza un rango máximo de $50-60 \%$ en casi todas las ventanas de tiempo desglosadas, y para casi todas las bandas EEG, lo que sugiere que, más allá de la dinámica correlacional que es posible encontrar entre series de datos originales EEG, provenientes de distintos pares de electrodos, existiría un segundo nivel de correlación, o meta-sincronía, relativa esta vez a la dinámica oscilatoria del balance caos/orden en el cerebro, y que ya no depende de los datos EEG propiamente tales, sino de la integración no-lineal de parámetros eléctricos cerebrales que en su conjunto definirían un espacio de fase probabilístico de tendencia global.

Al desglosar el estudio en diferentes ventanas temporales, es posible observar con más detalle la persistencia correlacional que presenta esta dinámica no-lineal del porcentaje de pares de electrodos meta-sincrónicos en el balance caos/orden, en el tiempo.

Los resultados permiten visualizar también la existencia de notables diferencias individuales en algunos sujetos (sujeto 2) con valores que se escapan significativamente de los encontrados en el resto de los participantes.

Aunque no se encontraron diferencias estadísticas significativas en el porcentaje de electrodos meta-sincrónicos y la banda EEG de estudio, los resultados muestran una tendencia porcentual a la baja desde la banda delta a la banda gamma (delta $=18.29 \%>$ theta $=14.84 \%>$ alpha $=12.69 \%>$ beta $=9.95 \%>$ gamma $=8.05 \%$ ) obtenidos a partir de los datos de la figura 5. Para corroborar este posible hallazgo en futuras investigaciones, extenderemos el tiempo de registro hasta los 5 minutos para obtener un $\mathrm{N}$ de porcentajes comparables que permita alcanzar los parámetros estadísticos en que se pueda hallar significancia.

\section{Conclusión}

El presente estudio exploratorio muestra la persistencia en el tiempo de procesos meta-sincrónicos no-lineales que obedecen a la dinámica del balance caos/orden global del cerebro, en condiciones de reposo, basal con ojos cerrados. Al mismo tiempo, podría sugerir la presencia activa de una meta-dinámica caos/orden global, la que podría modular los grados de libertad de procesos a escala local. Para explorar estas posibilidades se requeriría hacer experimentos de registro temporal más largo y en condiciones comparativas basal vs perceptual vs cognitiva, lo que podría arrojar más información respecto de cómo funciona el cerebro, en su dominio de existencia nolineal, para distintos estados de actividad y procesamiento cerebral.

Sería interesante estudiar estos fenómenos en otros grupos etarios (niños, adolescentes, adultos mayores, etc.) y en muestras femeninas, como una forma de contrastar los resultados aquí obtenidos. 


\section{Declaración de conflictos de interés}

Los autores declaran que en este estudio no existen conflictos de interés relevantes.

\section{Fuentes de financiamiento}

No existió una fuente de financiamiento particular para este informe científico.

\section{Referencias}

Bassingthwaighte J, Raymond G. Evaluating rescaled range analysis for time series. Annals of Biomedical Engineering, 1994; 2(4): 432-444.

Bear M, Connors B, Paradiso M. Neurociencia, la exploración del cerebro. $4^{\circ}$ ed. Madrid: Wolters Kluver, 2016.

Buzsaki G. Rhythms of the brain. London: Oxford University Press. 2006.

Corless M. Introduction to dynamic systems. Indiana: Purdue University. 2011

Díaz H, Córdova F, Cañete L, Palominos F, Cifuentes F, Sánchez C, et al. Order and chaos in the brain: fractal time series analysis of the EEG activity during a cognitive problem solving task. Procedia Computer Science, 2015; 55: 1410-1419. doi: 10.1016/j. procs.2015.07.135

Díaz H, Maureira F, Cohen E, Córdova F, Palominos F, Otárola J, et al. Individual differences in the order/chaos balance of the brain selforganization. Annals of Data Science, 2015; 2(3): 1-18. doi: 10.1016/j.procs.2017.11.378

Díaz H, Maureira F, Córdova F. Times series of closed and open eyes EEG conditions reveal differential characteristics in the temporality of linear and no-linear analysis domain. Procedia Computer Science, 2018; 139: 570-577. doi: 10.1016/j.procs.2018.10.208

Díaz H, Maureira F, Flores E, Córdova F. Intra e inter-hemispheric correlation of the order/ chaos fluctuation in the brain activity during a motor imagination task. Procedia Computer Science, 2018; 139: 456-463. doi: 10.1016/j.procs.2018.10.250

Díaz H, Maureira F, Flores G, Fuentes I, García F, Maertens P, et al. Moving correlations and chaos in the brain during closed eyes basal conditions. Procedia Computer Science, 2018; 139: 473-480. doi: 10.1016/j.procs.2018.10.248

Díaz H, Maureira F, Flores E, Gárate E, Muñoz S. Intra and inter-individual variability in the chaotic component and functional connectivity of the EEG signal in basal closed eyes condition. Procedia Computer Science, 2019; 162: 966-973. doi: 10.1016/j. procs.2019.12.077

Díaz H, Maureira F, Flores E, Cifuentes H, Córdova F. Synchronizing oscillatory chaos in the brain. Procedia Computer Science, 2019; 162: 982-989. doi: 10.1016/j. procs.2019.12.076

Díaz H, Maureira F, Otárola J, Rojas R, Alarcón O, Cañete L. EEG Beta band frequency domain evaluation for assessing stress and anxiety in resting, eyes closed, basal conditions. Procedia Computer Science, 2019; 162: 974-981.

Kumar J, Bhuvaneswari P. Analysis of electroencephalography (EEG) signals and its categorization-a study. Procedia Engineering, 2012; 38: 2525-2536. doi: 10.1016/j. proeng.2012.06.298

Layek G. An introduction to dynamical system and chaos. New York: Springer. 2015.

Maureira F. ¿Qué es la inteligencia? 1 ed. Madrid: Bubok Publishing, 2017.

Maureira F, Flores F. Electroencefalografía (EEG) y diversas manifestaciones del movimiento: una revisión del 2000 al 2017. EmásF, Revista Digital de Educación Física, 2018; 9(51): 48-63.

Michel C, Murray M. Towards the utilization of EEG as a brain imaging tool. NeuroImage, 2012; 61(2): 371-385. doi: 10.1016/j.neuroimage.2011.12.039 
Fernando Maureira Cid, Hernán Díaz-Muñoz, Marcelo Hadweh-Briceño, Elizabeth Flores-Ferro, Ángela Silva-Salse

Montero F, Moran F. Biofísica: procesos de auto-organización en biología. Madrid: EUDEMA. 1992

Pikovsky A, Rosenblum M, Kurths J. Synchronization: a universal concept in nonlinear sciences. $1^{\circ}$ ed. Cambridge: Cambridge University Press, 2001.

Raimundo M, Okamoto J. Application of Hurst Exponent $(\mathrm{H})$ and the R/S analysis in the classification of FOREX Securities. International Journal of Modeling and Optimization, 2018; 8(2): 116-124. doi: 10.7763/ijmo.2018.v8.635

World Medical Asociation. World Medical Association Declaration of Helsinki Ethical Principles for Medical Research Involving Human Subjects. JAMA, 2013; 310(20): 2191-2194. 